\title{
Erratum to: The approximation to a fixed point
}

Sibylla Priess-Crampe and Paulo Ribenboim

J. Fixed Point Theory Appl. 14 (2013), 41-53

DOI 10.1007/s11784-014-0157-4

In the statement of Theorem 3.5, the authors have noticed the following misprint and omission:

(1) in line 2 of this statement, " $a_{0} \in Y$ " should read " $a_{\iota^{\prime}} \in Y$ ",

(2) in line 3 of the statement, "Then $z \in \widehat{Y}$ and there exists ..." should read "Then $z \in \widehat{Y}$ and conversely, if $z \in \widehat{Y} \backslash Y$, then there exists ..."

Sibylla Priess-Crampe

Mathematisches Institut der Universität München

Theresienstr. 39

80333 München

Germany

e-mail: priess@math.lmu.de

Paulo Ribenboim

Department of Mathematics and Statistics

Queen's University

Kingston, Ontario K7L3N6

Canada

e-mail: mathstat@mast.queensu.ca

The online version of the original article can be found under doi: 10.1007/s11784-014-0157-4 